\title{
Probing of medium-scale traveling ionospheric disturbances using HF-induced scatter targets
}

\author{
N. F. Blagoveshchenskaya ${ }^{1}$, T. D. Borisova ${ }^{1}$, V. A. Kornienko ${ }^{1}$, I. V. Moskvin ${ }^{1}$, M. T. Rietveld ${ }^{2}$, V. L. Frolov ${ }^{3}$, \\ V. P. Uryadov ${ }^{3}$, L. M. Kagan ${ }^{4}$, Yu. M. Yampolski ${ }^{5}$, V. L. Galushko ${ }^{5}$, A. V. Koloskov ${ }^{5}$, S. B. Kasheev ${ }^{5}$, A. V. Zalizovski ${ }^{5}$, \\ G. G. Vertogradov ${ }^{6}$, V. G. Vertogradov ${ }^{6}$, and M. C. Kelley ${ }^{7}$ \\ ${ }^{1}$ Arctic and Antarctic Research Institute, St. Petersburg, Russia \\ ${ }^{2}$ EISCAT Scientific Association, Ramfjordbotn, Norway \\ ${ }^{3}$ Radiophysical Research Institute, Nizhny Novgorod, Russia \\ ${ }^{4}$ University of Western Ontario, London, ON, Canada \\ ${ }^{5}$ Institute of Radio Astronomy NAS, Kharkov, Ukraine \\ ${ }^{6}$ Rostov-on-Don State University, Rostov-on-Don, Russia \\ ${ }^{7}$ Cornell University, Ithaca NY, USA
}

Received: 7 November 2005 - Revised: 12 April 2006 - Accepted: 15 May 2006 - Published: 20 September 2006

Part of Special Issue "Twelfth EISCAT International Workshop"

\begin{abstract}
Experimental results from the Troms $\varnothing$ and Sura heating experiments at high and mid-latitudes are examined. It is shown that the combination of HF-induced target and bi-static HF Doppler radio scatter observations is a profitable method for probing medium-scale traveling ionospheric disturbances (TIDs) at high and mid-latitudes. HF ionospheric modification experiments provide a way of producing the HF-induced scatter target in a controlled manner at altitudes where the sensitivity to TIDs is highest. Bi-static HF Doppler radio scatter observations were carried out on the LondonTroms $\varnothing-$ St. Petersburg path in the course of a Troms $\varnothing$ heating experiment on 16 November 2004 when the pump wave was reflected from an auroral Es-layer. During Sura heating experiments on 19 and 20 August 2004, when the HF pump wave was reflected from the F2 ionospheric layer, multiposition bi-static HF Doppler radio scatter observations were simultaneously performed at three reception points including St. Petersburg, Kharkov, and Rostov-on-Don. Ray tracing and Doppler shift simulations were made for all experiments. A computational technique has been developed allowing the reconstruction of the TID phase velocities from multi-position bi-static HF Doppler scatters. Parameters of medium-scale TIDs were found. In all experiments they were observed in the evening and pre-midnight hours. TIDs in the auroral E-region with periods of about $23 \mathrm{~min}$ were traveling southward at speeds of $210 \mathrm{~m} / \mathrm{s}$. TIDs in the mid-latitudinal
\end{abstract}

Correspondence to: N. F. Blagoveshchenskaya (nataly@aari.nw.ru)
F-region with periods from 20 to 45 min travelled at speeds between 40 and $150 \mathrm{~m} / \mathrm{s}$. During quiet magnetic conditions the waves were traveling in the north-east direction. In disturbed conditions the waves were moving in the south-west direction with higher speeds as compared with quiet conditions. Possible sources for the atmospheric gravity waves at middle and high latitudes are discussed.

Keywords. Ionosphere (Ionosphere: Active experiments; Ionospheric disturbances) - Radio Science (Nonlinear phenomena)

\section{Introduction}

Internal atmospheric gravity waves (AGWs) in the neutral atmosphere manifest themselves in the ionosphere as traveling ionospheric disturbances (TIDs). AGWs and TIDs give an essential input to the dynamics and energetics of the upper atmosphere.

Many efforts have been invested in studying AGWs and TIDs both experimentally and theoretically (see, for example, Francis, 1975; Yeh and Liu, 1974; Hunsucker, 1982; Crowley and McCrea, 1988; Williams and Virdi, 1989; Williams, 1996; Williams et al., 1993; Hocke et al., 1996). TIDs are usually grouped into two classifications: largescale and medium-scale, depending on their wavelengths and periods. Large-scale TIDs have horizontal wavelengths greater than $1000 \mathrm{~km}$ and periods of about $30 \mathrm{~min}$ to $3 \mathrm{~h}$.

Published by Copernicus GmbH on behalf of the European Geosciences Union. 


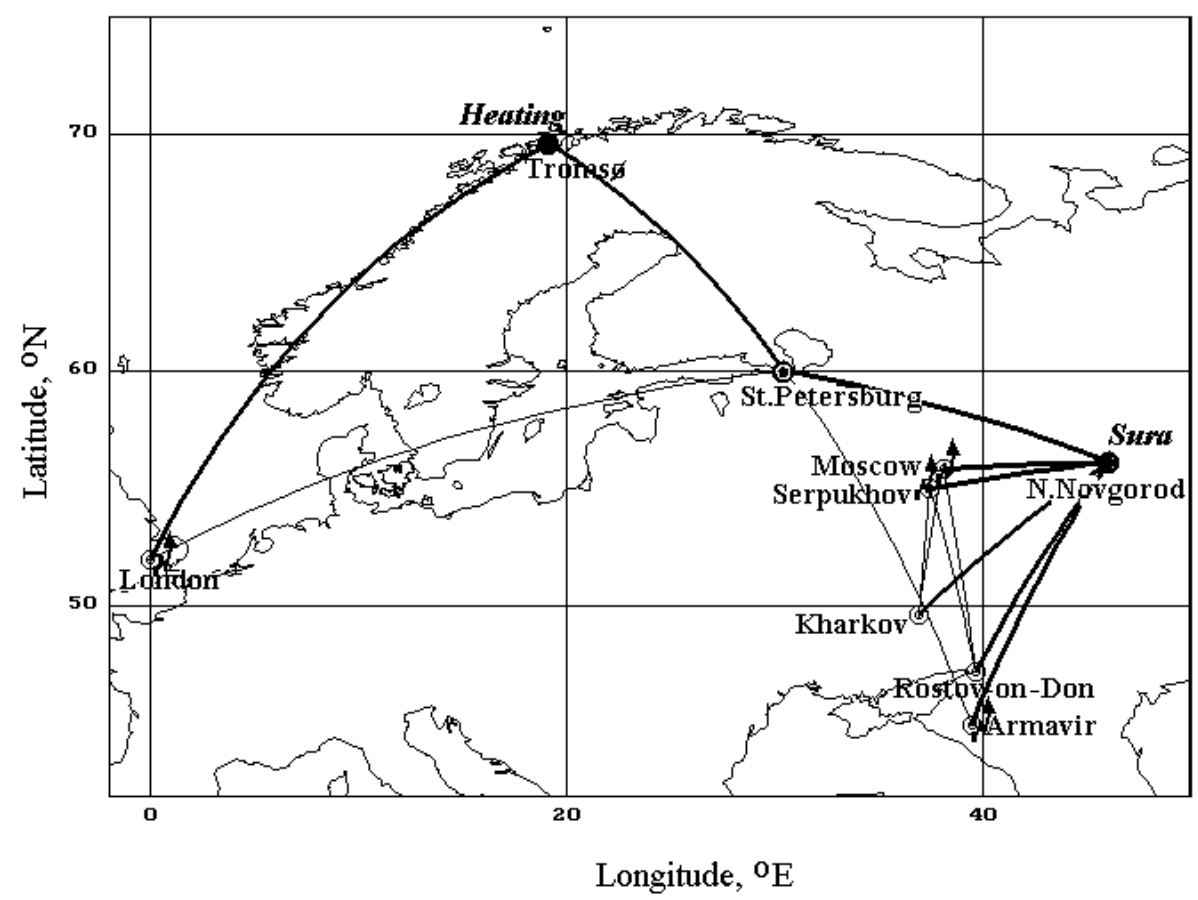

Fig. 1. A map indicating the experimental geometry. Bi-static HF Doppler radio scatter observations of AFAIs were carried out on the London-Troms $\emptyset$-St. Petersburg paths in the course of the Troms $\varnothing$ heating experiment in November 2004 and on the Serpukhov-SuraKharkov, Serpukhov (Moscow)-Sura-Rostov, and Armavir-Sura-St. Petersburg paths in the course of the Sura heating campaign in August 2004.

Medium-scale TIDs have periods of $15 \mathrm{~min}$ to $1 \mathrm{~h}$ and wavelengths of several hundred $\mathrm{km}$. The primary distinction between large- and medium-scale TIDs is in their phase velocities, which are, respectively, greater $(400-1000 \mathrm{~m} / \mathrm{s})$ and less $(100-250 \mathrm{~m} / \mathrm{s})$ than the thermospheric sound speed (Hunsucker, 1982).

Experimental studies of traveling ionospheric disturbances have been conducted using different diagnostic methods and instruments including ionosondes and trans-ionospheric sounding (Afraimovich et al., 1996), HF Doppler network (Crowley and McCrea, 1988), incoherent scatter radars (Kirchengast et al., 1995), coherent HF radars like SuperDARN (Bristow et al., 1994), and global GPS networks (Afraimovich et al., 2000).

The main body of TID studies is related to the large-scale traveling ionospheric disturbances. The medium-scale waves do not propagate as far as the large-scale waves and monitoring their progression would require a much greater spatial density of appropriate techniques than is currently available. The other problem arises from the effect of winds in the mesosphere on the propagation of medium-scale waves which have a much lower horizontal velocity than large-scale waves.

In the present paper, we propose the combination of HF ionospheric modification and bi-static HF Doppler radio scatters for identifying and studying traveling ionospheric disturbances. It is well known that the nonlinear interaction between a powerful HF radio wave and the ionospheric plasma leads to the excitation of artificial field-aligned irregularities (AFAIs). Their spatial scale is of the order of a few meters or tens of meters across and several kilometers along the magnetic field line. AFAIs act as an artificially produced target for the diagnostic HF radio waves which are then used to trace the gravity waves. We report experimental results related to probing medium-scale traveling ionospheric disturbances using HF-induced scatter targets at mid- and high latitudes. With bi-static HF Doppler scatter, the AFAIs are being studied at middle (Blagoveshchenskaya and Troshichev, 1996; Yampolski et al., 1997; Blagoveshchenskaya et al., 1998a) and high latitudes (Blagoveshchenskaya et al., 1998b, 1999, 2006; Rietveld et al., 2003). The proposed observational technique was used for the first time to identify the medium-scale TIDs. This technique offers several advantages over the HF Doppler network where HF signals are propagating from the transmitter to the receiver along a great circle path (direct signals). The sensitivity of HF Doppler network to a TID depends on the frequency transmitted and increases dramatically if this is close to the F-region critical frequency. Moreover, significant errors may be introduced into the horizontal velocity estimates if Doppler reflection heights for different diagnostic radio paths are not equal. HF ionospheric modification experiments provide a way of 
producing a HF heater-induced target in a controlled way. The spatial features of the target are known. The altitude of the target is close to the reflection level of the powerful HF radio wave. Usually the HF heater frequency is chosen in the vicinity of the maximum plasma frequency where the sensitivity to the TIDs is highest. In addition, the diagnostic signals from different locations are scattered from the common HF heater-induced target, thereby allowing TID parameters to be obtained with more accuracy.

Our analyses cover a number of days which were part of a coordinated Sura heating campaign in August 2004 at middle latitudes and a Troms $\varnothing$ heating campaign in November 2004 at high latitudes. In the course of the Troms $\varnothing$ heating campaign the HF heater-induced target was produced in the auroral E-region of the ionosphere. Therefore, the mediumscale TIDs were observed in the sporadic Es-layer. Note that previous studies have been related to the TIDs in the F-region of the ionosphere. The behaviour, parameters, and possible sources of medium-scale TIDs are then discussed.

\section{Experimental arrangement}

The experiments reported here were carried using the EISCAT HF heating facility, as well as the Sura heating facility. The EISCAT HF heating facility (geographical coordinates $69.6^{\circ} \mathrm{N} ; 19.2^{\circ} \mathrm{E}, \mathrm{L}=6.2$ ), located near Troms $\varnothing$, Norway (Rietveld et al., 1993), was used for the modification of the auroral ionosphere. The experiment was conducted on 16 November 2004 in the pre-midnight hours. The HF facility was operated at $4040 \mathrm{kHz}$, using o-mode polarisation. The low gain antenna (phased array 2) was utilized, resulting in an effective radiated power (ERP) of $190 \mathrm{MW}$. The HF transmission scheme of 2 min on, 1 min off was used with the HF antenna beam pointed $9^{\circ}$ south of zenith.

Observations from the Sura heating campaign were carried out on 19 and 20 August 2004 in the evening and premidnight hours. The Sura heating facility $\left(56.13^{\circ} \mathrm{N} ; 41.6^{\circ} \mathrm{E}\right)$ is located at Vasilsursk near N. Novgorod, Russia. On 19 August 2004 an ordinary mode pump wave was radiated at $4300 \mathrm{kHz}$ in the vertical direction with a $5 \mathrm{~min}$ on, $5 \mathrm{~min}$ off transmission scheme. On 20 August the Sura facility was operated at $4785 \mathrm{kHz}$ radiating o-mode polarisation. An $\mathrm{HF}$ transmission scheme of $10 \mathrm{~min}$ on, $5 \mathrm{~min}$ off was used. The heater antenna beam was tilted by $12^{\circ}$ to the south of zenith. The effective radiated power was $150 \mathrm{MW}$.

In the course of all experiments reported here, bi-static HF Doppler radio scatter observations were used for diagnostics of AFAIs.

The geometry of the Troms $\varnothing$ and Sura heating experiments is shown in Fig. 1. On 16 November 2004 observations were carried out on the London-Troms $\varnothing-$ St. Petersburg path at an operational frequency of $12095 \mathrm{kHz}$. The reception of diagnostic waves scattered by AFAIs above Troms $\emptyset$ was made with a Doppler spectral method in St. Petersburg at a dis-
Table 1. Locations and geographical coordinates of transmitting and receiving sites.

\begin{tabular}{|l|l|l|l|l|l|}
\hline \multirow{2}{*}{$\begin{array}{l}\text { Locations of transmitting } \\
\text { and receiving sites }\end{array}$} & \multicolumn{2}{|l|}{ Coordinates } & Operational & Distance to Sura, \\
\cline { 2 - 4 } & Lat. N & Long. E & frequency, kHz & $\mathrm{km}$ \\
\hline Transmitter & Moscow & 55.8 & 38.3 & 14996 & 490 \\
& Serpukhov & 55.0 & 37.5 & 11630 & 550 \\
& Armavir & 45.0 & 39.5 & 15455 & 1320 \\
\hline Receiver & St. Petersburg & 60.5 & 30.5 & & 1030 \\
& Kharkov & 49.6 & 36.9 & & 945 \\
& Rostov & 47.2 & 39.7 & & 1080 \\
\hline
\end{tabular}

tance of about $1200 \mathrm{~km}$ from Troms $\varnothing$; the receiving antenna was directed toward Troms $\varnothing$. Note that at the geographical location of St. Petersburg, the geometry for bi-static scatter is satisfied for the Troms $\varnothing$ HF heater, as well as for the Sura facility. Consequently, during the Sura heating campaign in August 2004 bi-static scatter observations were carried out near St. Petersburg with the use of the same HF Doppler equipment. For the August heating experiments a new antenna system was installed near St. Petersburg that pointed to the Sura heating facility. The spectral processing was carried out by using a windowed (Hanning) fast Fourier transform (FFT). In the course of the Sura and Troms $\varnothing$ heating experiments described here the sample rate of $102 \mathrm{~Hz}$ allowed a spectral analysis bandwidth of $51 \mathrm{~Hz}$. A specified number of 512 FFT coefficients provided a frequency resolution of $0.1 \mathrm{~Hz}$. Each segment used for the FFT was overlapped by $50 \%$ which yields the production of a Doppler spectrum every $5 \mathrm{~s}$. The distinctive feature of the Sura heating campaign in August 2004 is that bi-static HF Doppler radio scatter observations were simultaneously carried out at three receiving sites including St. Petersburg, Kharkov, and Rostov-on-Don. At Kharkov the reception of diagnostic signals scattered from AFAIs was made with the east-west (EW) and north-south (NS) beams of the UTR 2 radio telescope phased array. It is one of the largest research antennas in the decametric wavelengths (frequencies from 10 to $30 \mathrm{MHz}$ ). The phased array has a T-shaped configuration and consists of two subarrays extended in the north-south (NS) and east-west (EW) directions. Their lengths are about 2 and $1 \mathrm{~km}$, respectively (Braude et al., 1978). At Kharkov and Rostov-on-Don the spectral analysis bandwidth of $20 \mathrm{~Hz}$ was used. A specified number of 256 FFT coefficients allowed a frequency resolution of about $0.1 \mathrm{~Hz}$. The locations and geographical coordinates of the transmitting and receiving sites employed in the course of the Sura heating campaign are given in Table 1. 

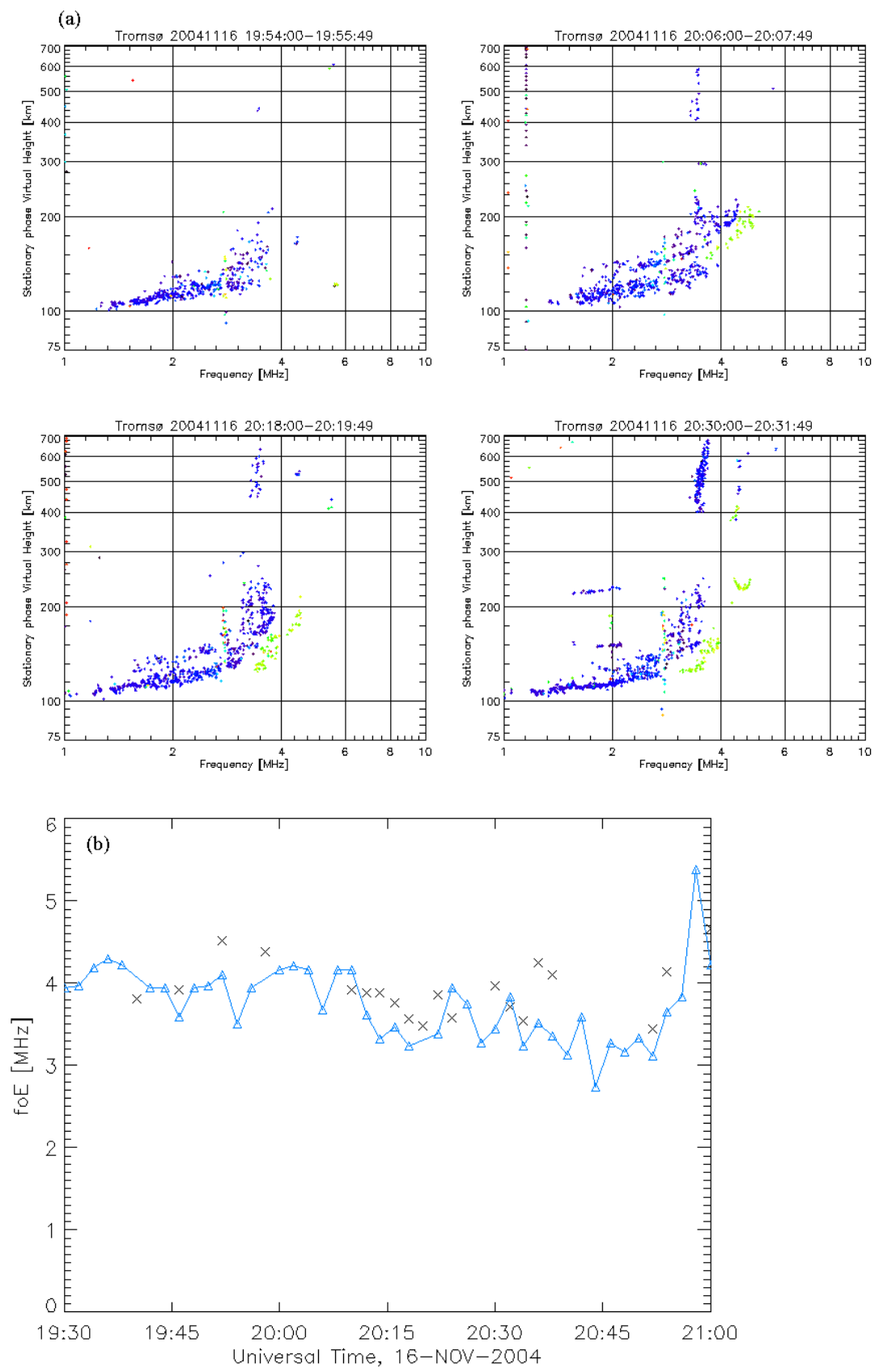

Fig. 2. Ionograms obtained during Troms $\varnothing$ HF pumping experiment on 16 November 2004. Blue echoes are O mode and green echoes $X$ mode (a). Temporal variations of $f o \mathrm{E}$ (blue triangles joined by straight lines) and $f_{o} \mathrm{~F} 2$ (black crosses) on 16 November 2004 from 19:30 to 21:00 UT are shown in (b).

\section{Observational results at auroral latitudes}

The EISCAT heating experiment was conducted on 16 November 2004 from 19:30 to 21:00 UT under moderate magnetic activity. Troms $\varnothing$ dynasonde data during the experiment are presented in Fig. 2. Ionograms from the Troms $\varnothing$ dynasonde (see Fig. 2a) showed that, during the experiment, an intense sporadic Es-layer with critical fre- quencies between 3.8 and $4.1 \mathrm{MHz}$ was present. A plot of $f o \mathrm{E}$ (blue triangles joined by straight lines) and $f o \mathrm{~F} 2$ (black crosses) versus time is shown in Fig. 2b. These were obtained by an automatic echo processing program, DSND, written by J. W. Wright (http://www.ngdc.noaa.gov/ stp/IONO/Dynasonde/tutorial/tutorial.html). As can be seen from Fig. 2, in most cases artificial field-aligned irregularities were produced in the auroral E-region. There is sometimes 


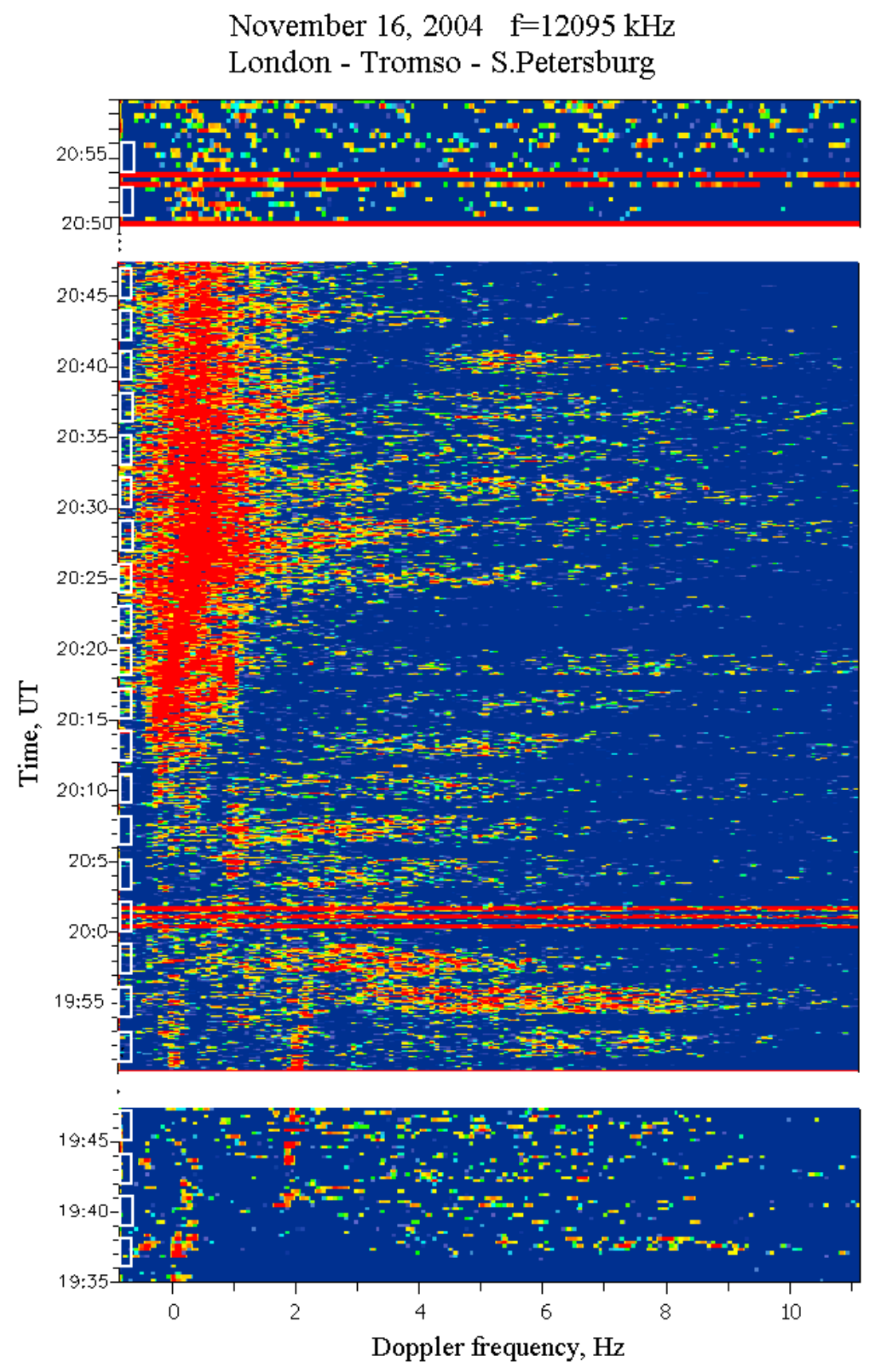

Fig. 3. Dynamic Doppler spectra of HF diagnostic signals on the London-Troms $\emptyset-$ St. Petersburg path on 16 November 2004 from 19:3521:00 UT at operational frequency $f=12095 \mathrm{kHz}$. The HF heater beam was tilted by $9^{\circ}$ to the south. The direct signals propagating from the transmitter to the receiver along a great circle path correspond to zero Doppler shifts. The intervals when the EISCAT Troms $\varnothing$ HF heating facility was turned on are marked by bars on the time axis.

an F-region visible at about the same frequency as the Eslayer critical frequency.

Figure 3 displays dynamic Doppler spectra (sonogram) of HF diagnostic signals on the London-Troms $\varnothing-$ St. Petersburg path obtained on 16 November 2004 from 19:35 to 21:00 UT for the radio scatter operational frequency of $12095 \mathrm{kHz}$. As can be seen from Fig. 3, strong field-aligned scattered sig- nals appear after the heater is turned on and disappear after it is turned off. They are recorded as additional tracks shifted between about 1.5 and $8 \mathrm{~Hz}$ from that of the direct signal propagating from the transmitter to receiver along a great circle path corresponding to the $0 \mathrm{~Hz}$ Doppler frequency. Note that striations were excited even when the HF heater frequency was slightly larger than the critical frequency ( $f o \mathrm{E}$, 

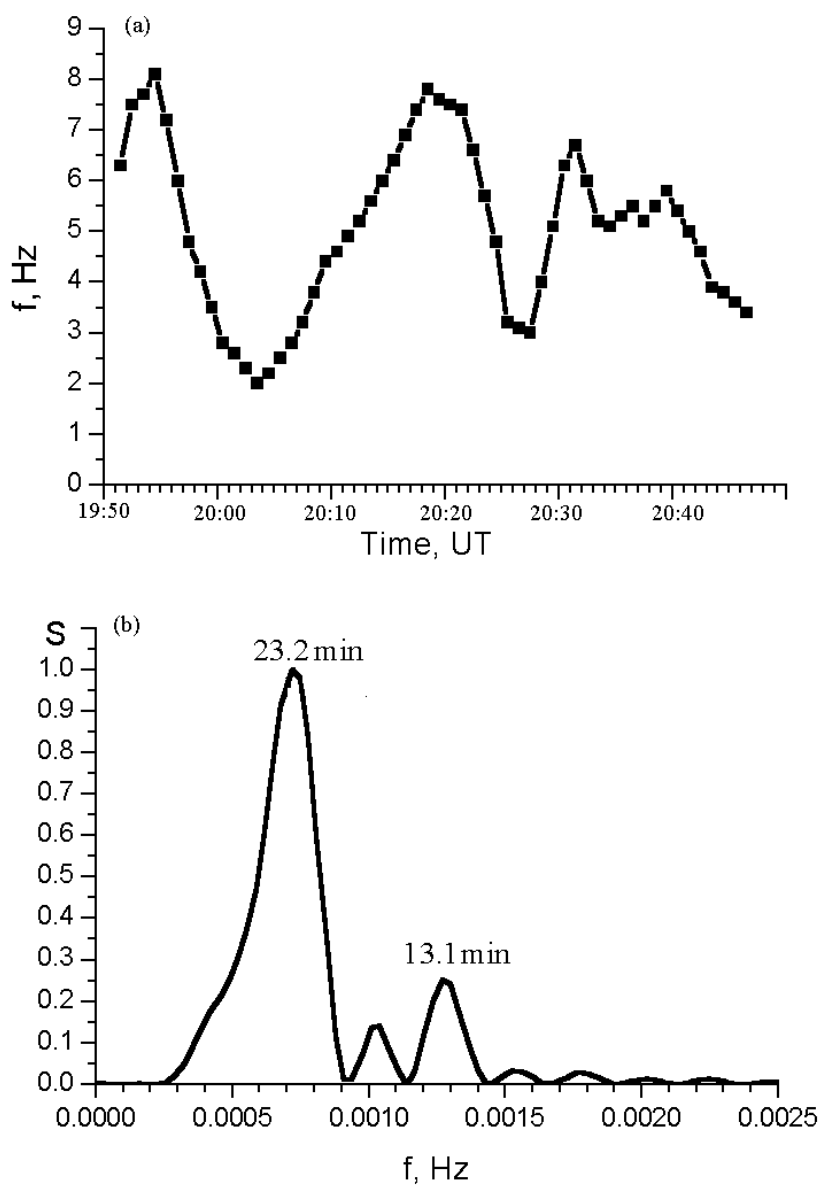

Fig. 4. Median values of Doppler frequency shifts averaged on the 1-min time interval for diagnostic signals scattered from AFAIs on the London-Troms $\varnothing$-St. Petersburg path on 16 November 2004 from 19:50-20:50 UT (a); the spectral density of the Doppler shift variations for the same event (b).

$f o \mathrm{~F} 2$ ), because it is the upper-hybrid frequency that is important, which is about $200 \mathrm{kHz}$ above $f o \mathrm{E}, f_{o \mathrm{~F}} 2$. The scattered signals are characterised by a wide spectral broadening and wave-like variations in the median Doppler shifts of signals scattered from AFAIs on the positive part of the Doppler sonogram. To find their periods we have made a spectral analysis of the median Doppler frequency shifts $f_{d}$ of the scattered signals through the analysed time interval. The median values of $f_{d}$ averaged a 1-min time interval in the course of the Troms $\varnothing$ heating experiment on 16 November 2004 are shown in Fig. 4a. During the 1 min heater-off intervals, an interpolation of $f_{d}$ values was employed. As can be seen from Fig. 4a, the maximum peak-to-peak of the wave reached $6 \mathrm{~Hz}$. Figure $4 \mathrm{~b}$ presents the calculated power density spectra. It was calculated from the auto-correlation function of $f_{d}$ temporal variations using the Wiener-Kinchine theorem (Jenkins and Watts, 1968). The main period of the wave was about $23 \mathrm{~min}$, falling within the range of the medium-scale traveling ionospheric disturbances.
Simulation results of the Doppler frequencies shifts of scattered signals for the London-Troms $\varnothing-$ St. Petersburg path intimate that the velocity component in the north-south direction is crucial in the Doppler frequency shift values (Borisova et al., 2002). The positive Doppler frequency shifts $\left(+f_{d}\right)$ correspond to the southward motions of AFAIs in the HF heater-induced scattering volume. Therefore, in the course of the experiment on 16 November 2004, the passage of the traveling ionospheric disturbance was in the southward direction.

It is possible to estimate the velocity $V$ of a medium-scale TID from the expression (Afraimovich, 1982)

$V=2 \pi h_{0} \sqrt{M / 2 h_{0}} / T$,

where $M=T\left(f_{d \max }-f_{d \min }\right) \lambda / 2 \pi ; T$ is the period of the wave, $\left(f_{d \text { max }}-f_{d \text { min }}\right)$ is the amplitude of wave from HF Doppler data, $\lambda$ is the wavelength of the diagnostic transmitter, and $h_{0}$ is the altitude of AFAIs.

In our case taking into account the experimental values of $T=23 \mathrm{~min},\left(f_{d \max }-f_{d \min }\right)=6 \mathrm{~Hz}, \lambda=25 \mathrm{~m}$, and $h_{0}=120 \mathrm{~km}$ the velocity of the TID is about $V=210 \mathrm{~m} / \mathrm{s}$.

\section{Mid-latitude medium-scale TIDs}

\subsection{Observations}

The SURA heating experiment on 19 August 2004 was conducted under quiet magnetic conditions $\left(K_{p}=1\right)$. The day of 20 August 2004 was geomagnetically disturbed with $K_{p}=3$ 4. Ionograms from the ionosonde at Sura showed that the critical frequencies of the F2-layer exceeded the pump frequencies used in the experiments. There also was no blanketing sporadic Es-layer. We therefore conclude that the F2layer was actually the region where the powerful HF radio waves were reflected. On 19 August 2004 very strong signals scattered from AFAIs were observed from 18:31 UT. However, wave-like variations in the Doppler frequency shifts revealed themselves only from 20:00 to 20:30 UT. At 20:30 UT the Sura heating facility was turned off.

Figure 5 depicts dynamic Doppler spectra (sonograms) of HF diagnostic signals on 19 August 2004 from 20:00 to 20:30 UT. Observations were carried out on the ArmavirSura-St. Petersburg path at the operational frequency of $15455 \mathrm{kHz}$ and on the Serpukhov-Sura-Kharkov path at $f=11630 \mathrm{kHz}$ with the east-west (EW) and north-south (NS) beams of the UTR-2 phased array. The direct signals for every path propagating from the transmitter to the receiver along a great circle path correspond to the zero Doppler shifts.

Similar to the event on 16 November 2004, the mediumscale traveling ionospheric disturbances on 19 August 2004 can be identified from the Doppler sonograms by wave-like variations in the Doppler frequency shifts of diagnostic signals scattered from AFAIs. From Fig. 5 we can see that the 

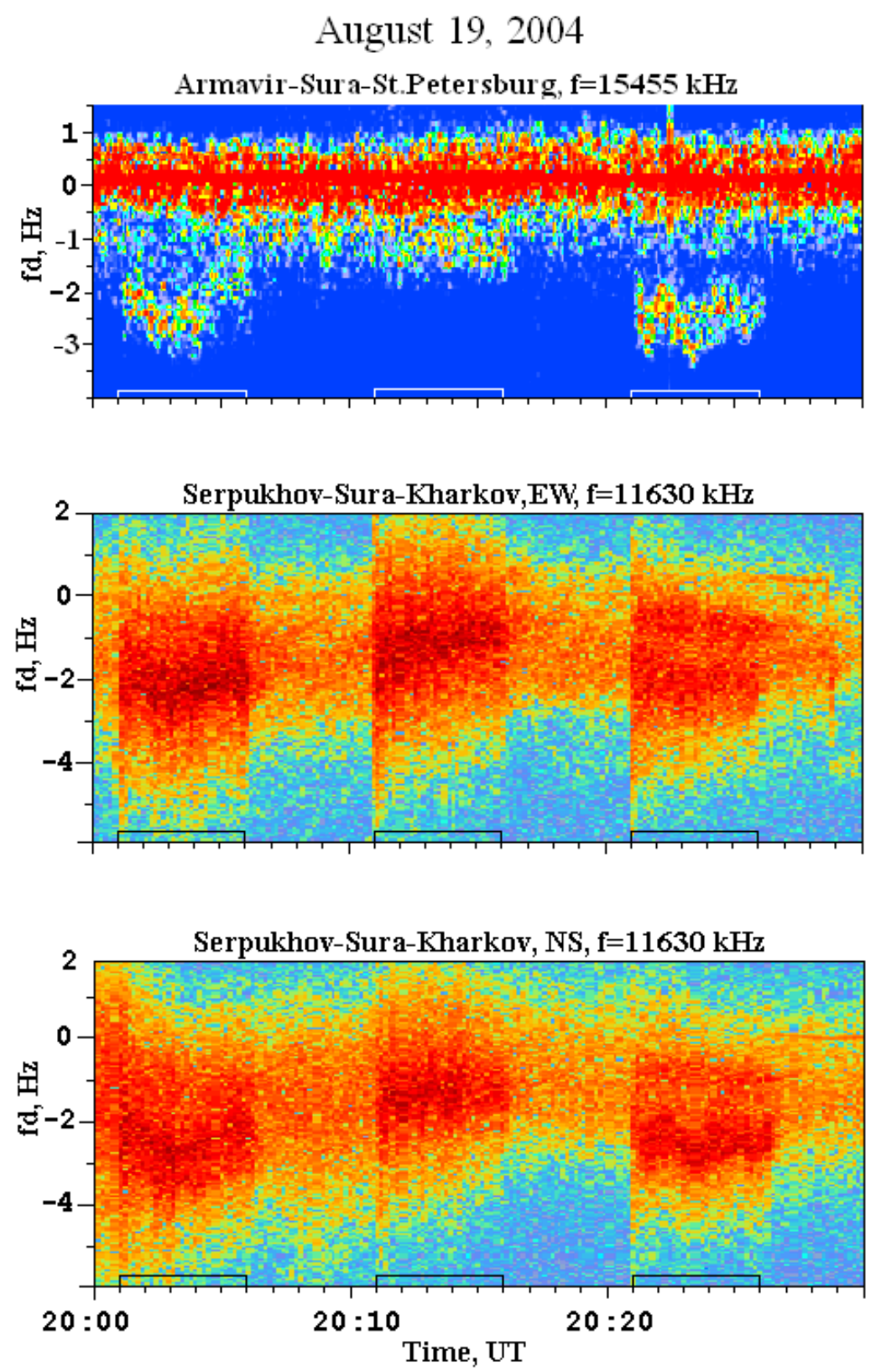

Fig. 5. Dynamic Doppler spectra of HF diagnostic signals on 19 August 2004, from 20:00 to 20:30 UT. Observations were carried out on the Armavir-Sura-St. Petersburg path, $f=15455 \mathrm{kHz}$, and on the Serpukhov-Sura-Kharkov path, $f=11630 \mathrm{kHz}$ with the east-west (EW) and north-south (NS) beams of the UTR-2 phased array. The direct signals for every path propagating from the transmitter to the receiver along a great circle path correspond to zero Doppler shifts. The intervals when the Sura HF heating facility was turned on are marked by bars on the time axis. The ordinary mode pump wave was transmitted at $4300 \mathrm{kHz}$ in the vertical direction.

period of the wave process was about $20 \mathrm{~min}$ and its magnitude did not exceed $2 \mathrm{~Hz}$.

On 20 August 2004 the signals scattered from AFAIs were detected from 16:01 UT. At 19:26 UT the Sura facility was turned off. Dynamic Doppler spectra of HF diagnostic signals on 20 August 2004 from 18:20 to 19:30 UT are presented in Fig. 6. Observations were conducted simultaneously on the Armavir-Sura-St. Peters- burg path, $f=15455 \mathrm{kHz}$, Serpukhov-Sura-Kharkov path, $f=11630 \mathrm{kHz}$, and Serpukhov(Moscow)-Sura-Rostov path, $f=14996 \mathrm{kHz}$ and $1160 \mathrm{kHz}$. Again, in this event we also observed well defined wave signatures in the Doppler frequency shifts of scattered signals which can be associated with medium-scale traveling ionospheric disturbances. Their main period was about $45 \mathrm{~min}$ and the magnitude from Doppler measurements reached $4 \mathrm{~Hz}$ peak-to-peak. It is 

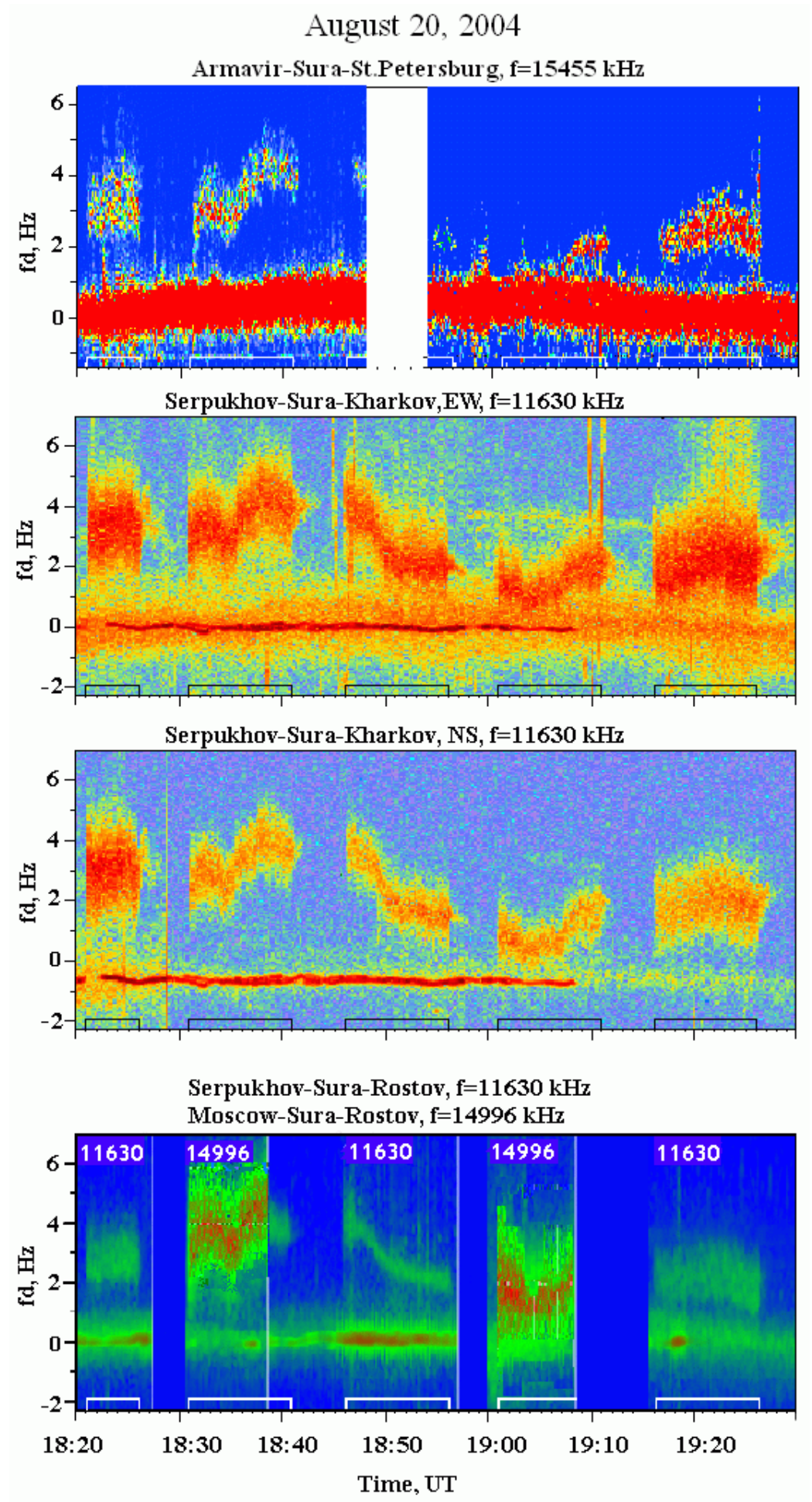

Fig. 6. Dynamic Doppler spectra of HF diagnostic signals on 20 August 2004 from 18:20 to 19:30 UT. Observations were carried out on the Armavir-Sura-St. Petersburg path, $\mathrm{f}=15455 \mathrm{kHz}$, Serpukhov-Sura-Kharkov path, $f=11630 \mathrm{kHz}$ with EW and NS beams of the UTR-2 phased array, and Serpukhov (Moscow)-Sura-Rostov path, $f=11630$ (14996) kHz. The direct signals for every path propagating from the transmitter to the receiver along a great circle path correspond to zero Doppler shifts. The intervals when the Sura HF heating facility was turned on are marked by bars on the time axis. The ordinary mode pump wave was transmitted at $4785 \mathrm{kHz}$. The HF heater beam was tilted by $12^{\circ}$ to the south. 

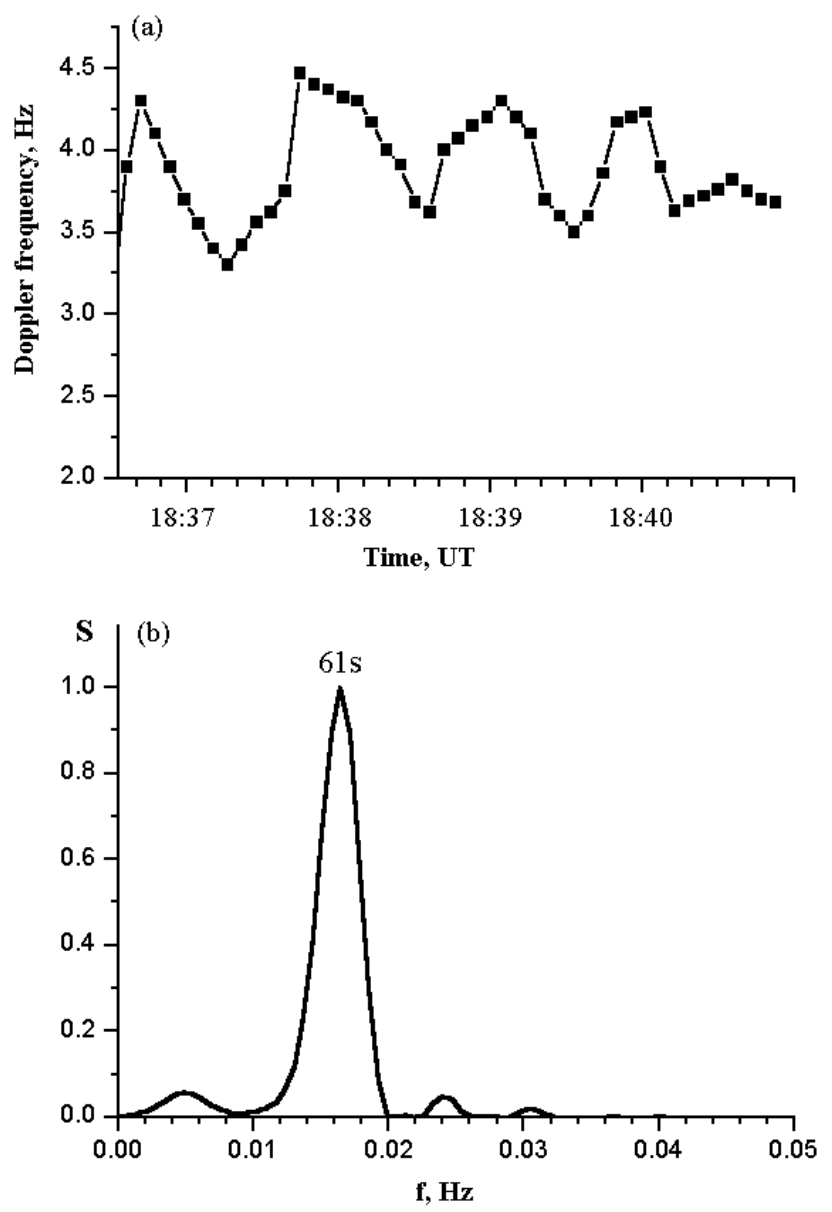

Fig. 7. Median values of Doppler frequency shifts for signals scattered from AFAIs on the Armavir-Sura-St. Petersburg path on 20 August 2004 during the Sura heater-on period from 18:36:30 to 18:41 UT (a); the spectral density of the Doppler shift variations for the same event (b).

interesting that together with long-period wave variations in $f_{d}$ with a period of $T=45 \mathrm{~min}$, there were the shorter periodic processes.

The consideration of experimental data, obtained simultaneously at three receiving sites in the course of experiments on 19 and 20 August 2004, has shown the close correlation between $f_{d}$ variations in the signals scattered from a common volume on all radio paths. The cross-correlation coefficients between different pairs of signals were not less than 0.9 . The relatively slow $f_{d}$ variations, associated with medium-scale traveling ionospheric disturbances, were sometimes modulated by shorter-period $f_{d}$ oscillations. For example, Fig. 7 shows the behaviour of the $f_{d}$ median values on the Armavir-Sura-St. Petersburg path on 20 August 2004 during the heater-on period from 18:36:30-18:41 UT (a) and the spectral density of these $f_{d}$ variations (b). The spectral procedure was the same as discussed in Sect. 3, but 5-s data were employed. From Fig. $7 \mathrm{~b}$ one can see the main period

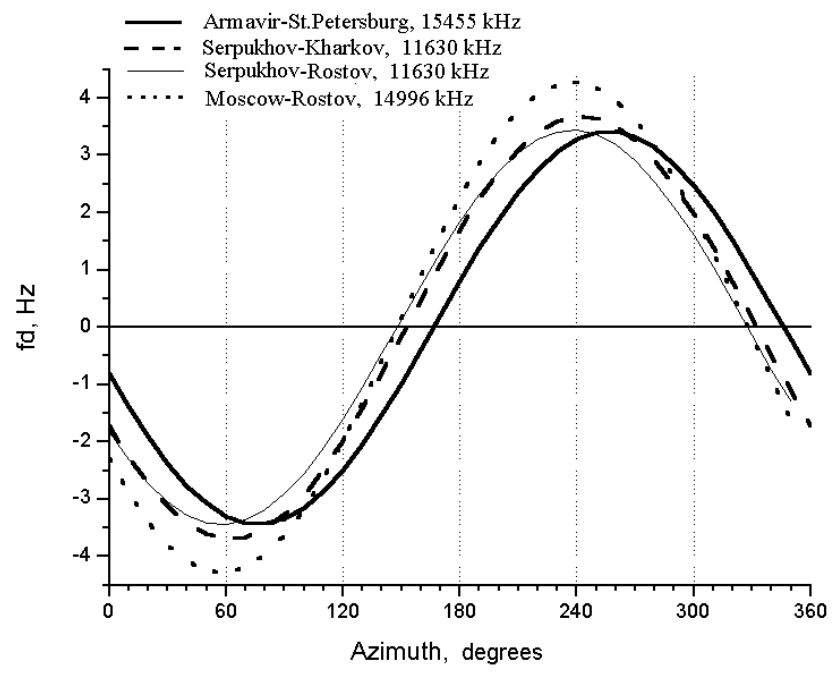

Fig. 8. Simulated values of Doppler frequency shifts fd versus the direction of the velocity in the HF heater-induced target for its magnitude of $50 \mathrm{~ms}^{-1}$ on the Armavir-Sura-St. Petersburg path at $f=15455 \mathrm{kHz}$, Serpukhov-Sura-Kharkov path at $f=11630 \mathrm{kHz}$, and Serpukhov (Moscow)-Sura-Rostov path at $f=11630(14996) \mathrm{kHz})$.

in $f_{d}$ variations of $61 \mathrm{~s}$. It corresponds to the ULF activity in the range of the $P c 3-4$ magnetic pulsations.

\subsection{Simulation results}

For analysis of multi-position bi-static HF Doppler observations, a simulation of the relationship between Doppler frequency shifts and the direction of velocities in the scattering volume was made. Figure 8 shows the behaviour of the simulated $f_{d}$, depending on the velocity direction on the ArmavirSura-St. Petersburg path $(f=15455 \mathrm{kHz})$, as well as on the Serpukhov-Sura-Kharkov and Serpukhov (Moscow)-SuraRostov paths at $11630(14996) \mathrm{kHz}$. The simulation was made by using the model described by Borisova et al. (2002). From Fig. 8 we can see that positive and negative values of $f_{d}$ on all radio paths correspond to the south-west and northeast directions of the AFAI motions, respectively.

Viewing line-of-sight Doppler frequency shifts from different angles allows the reconstruction of the vector phase velocity of the TID in the HF heater-induced volume. The amplitude and direction of velocities from multi-position bistatic Doppler scatter observations can be found as follows.

The projection of the velocity $V_{\Delta k}$ on the vector $\Delta \boldsymbol{k}(\Delta \boldsymbol{k}$ is the difference between wave vectors of the incident $\boldsymbol{k}_{0}$ and scattered $\boldsymbol{k}_{S}$ waves, $\Delta \boldsymbol{k}=\boldsymbol{k}_{S}-\boldsymbol{k}_{0}$ ) is determined by the expression (Gershman et al., 1984)

$V_{\Delta \mathrm{k}}=\left(f_{\mathrm{D}} / f\right) \cdot(c / 2 \sin (\theta / 2))$,

where $f_{\mathrm{D}}$ is Doppler frequency shift, $f$ is the operational frequency, $\theta$ is the scattering angle. 
August 19, 2004

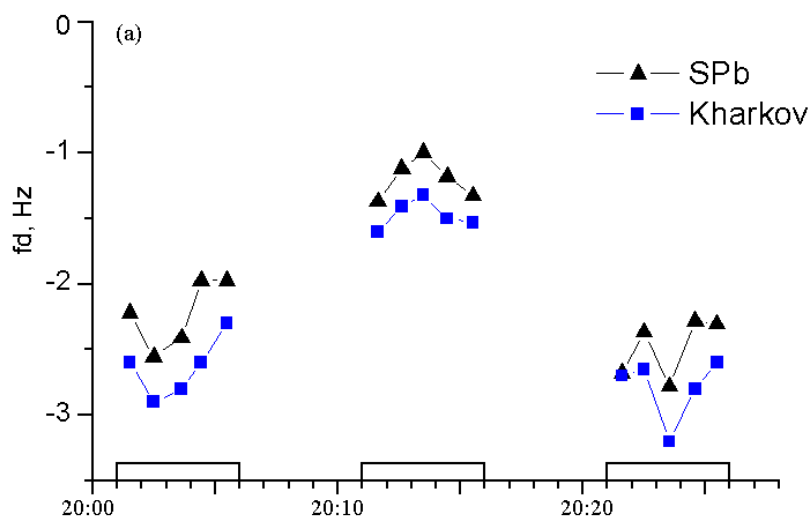

(b)

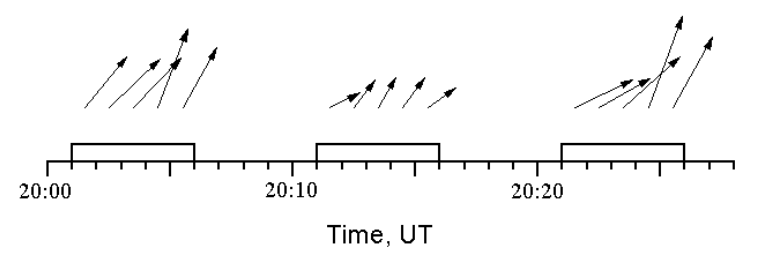

Fig. 9. Median values of Doppler frequency shifts averaged on the 1-min interval for the HF diagnostic signals scattered from AFAIs on the Armavir-Sura-St. Petersburg and Serpukhov-Sura-Kharkov paths in course of the Sura heating experiment on 19 August 2004 (a); simulated vectors of the TID phase velocities for the same event (b).

The simultaneous measurements of Doppler frequency shifts on two diagnostic paths, $f_{D 1}$ and $f_{D 2}$, as well as the values of scattering angles, $\theta_{1}$ and $\theta_{2}$, calculated from spherical geometry allows the projections of velocities $V_{1}$ and $V_{2}$ to be calculated using Eq. (2). Thereafter, the magnitude $V$ and the direction $A z_{V}$ of the vector phase velocity of the TID are found as follows

$V=V_{2} / \cos \{\operatorname{arctg}[(\cos \alpha-\beta) / \sin \alpha]\}$,

$A z_{\mathrm{V}}=A z_{\mathrm{k} 1}-\operatorname{arctg}[(\cos \alpha-\beta) / \sin \alpha]$,

where $\beta=V_{1} / V_{2} ; \alpha=\left|A z_{k 1}-A z_{k 2}\right|$ is the angle between the azimuths of the $\Delta \boldsymbol{k}_{1}$ and $\Delta \boldsymbol{k}_{2}$ vectors for the first and second paths, respectively.

Results from the reconstruction of the vector phase velocity of the TID for the experiments on 19 and 20 August 2004 are displayed in Figs. 9 and 10, correspondingly. From the reconstruction results we can find that, in the mid-latitude F-region in the course of the experiment on 19 August 2004, the waves were traveling in the north-east direction at a speed of about $40-80 \mathrm{~m} / \mathrm{s}$. On 20 August 2004 there were three available signal paths which allowed us to compute the three different velocity estimates using the three different pairs of paths. Calculation results are shown in Figs. 10b, c, and d. One can see the satisfactory agreement between velocity estimates on the three different pairs of paths. During the experiment on 20 August 2004 the waves were traveling in the south-west direction at a speed of about $60-150 \mathrm{~m} / \mathrm{s}$.

\section{Discussion}

The identification of sources for medium-scale AGWs responsible for traveling ionospheric disturbances is complicated because there are both tropospheric and ionospheric sources. Medium-scale AGWs can be induced by a multiplicity of sources at different altitudes, ranging from Joule heating and Lorentz forcing in the auroral electrojets to the progression of the solar terminator, perturbations in the jet-streams, thunderstorms, earthquakes, volcanoes, rocket launches (Williams, 1996; Bristow et al., 1994; Hocke et al., 1996; Cheng and Huang, 1992; Jacobson and Carlos, 1994). Moreover, multiple gravity wave packets from different sources may exist simultaneously. This adds complexity to the problem of source identification. As a result, it is often impossible to determine the source of medium-scale waves unambiguously. At auroral latitudes in the course of the Tromsø heating experiment on 16 November 2004, the observed traveling ionospheric disturbance with a period of about $23 \mathrm{~min}$ was moving at speeds of $210 \mathrm{~m} / \mathrm{s}$. These values are typical for medium-scale TIDs. The waves were traveling southward. It should be noted that these medium-scale TIDs were observed in the auroral E-region, because the HFinduced target certainly being produced in the sporadic Eslayer. This point is interesting in itself, because of the large body of previous studies that has been related to TIDs in the F-region of the ionosphere.

Most high-latitude medium-scale AGWs are observed to propagate equatorward and are thought to be generated by dayside auroral sources. Samson at al. (1990) found that the source of the AGWs detected by the Goose Bay HF radar was located near the dayside convection-reversal boundary in the E-region of the ionosphere.

Data from the IMAGE magnetometer network showed that the analysed time interval on 16 November 2004 is characterised by a change from the eastern electrojet to the western one. It corresponds to the nightside convection-reversal boundary. We suggest that the most plausible source of the AGWs, responsible for the medium-scale traveling ionospheric disturbances in the auroral E-region, is the nightside convection-reversal boundary.

Mid-latitude medium-scale TIDs in the ionospheric F-region were detected from multi-position bi-static HF scatter observations in the course of the Sura heating campaign in August 2004. They were observed in the evening and pre-midnight hours. By combining the observations with the results of simulation the parameters of medium-scale TIDs 


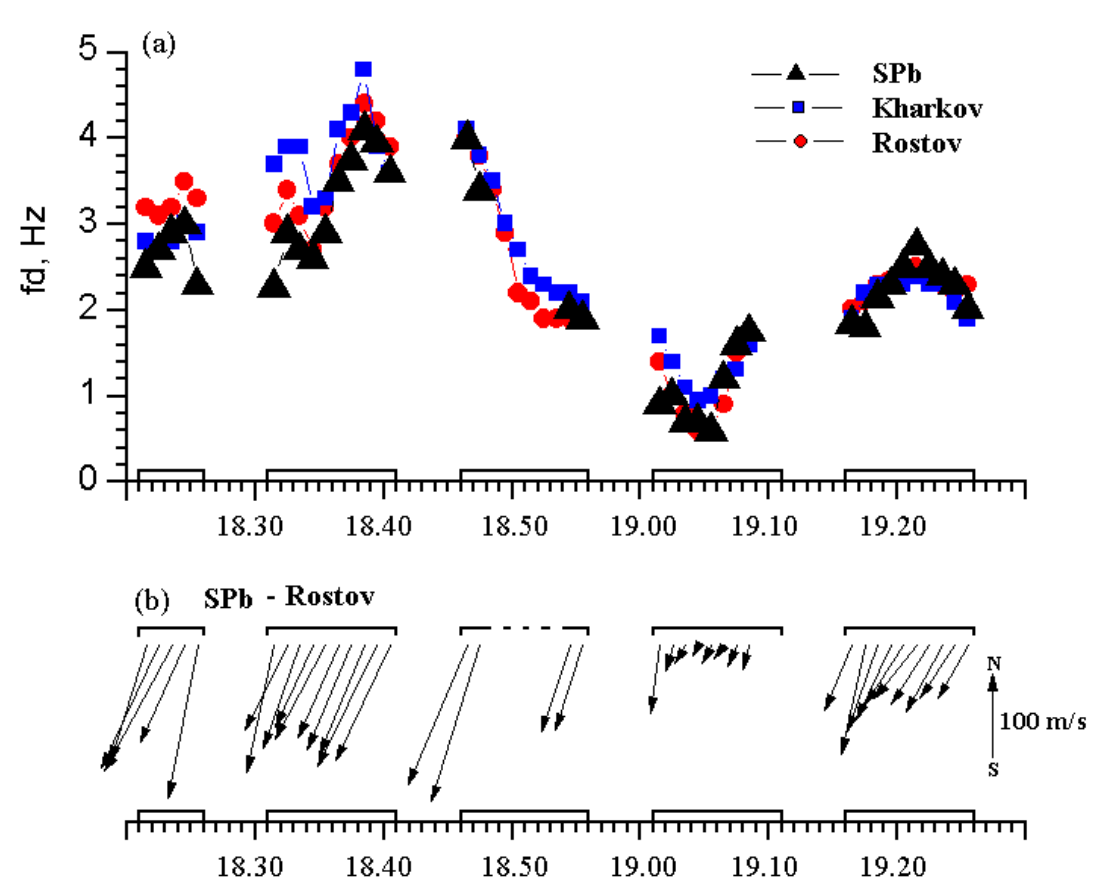

(c) SPb - Kharkov

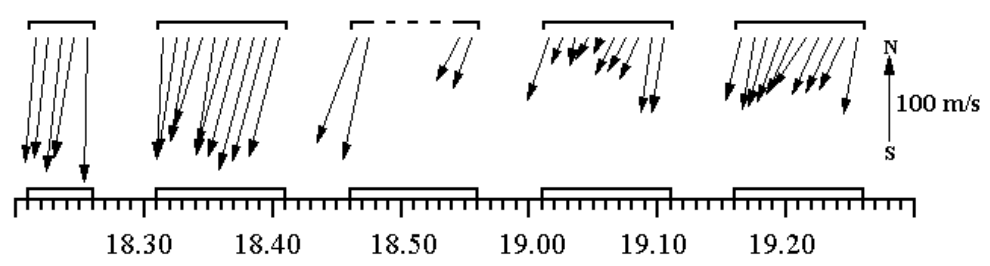

(d) Kharkov - Rostov

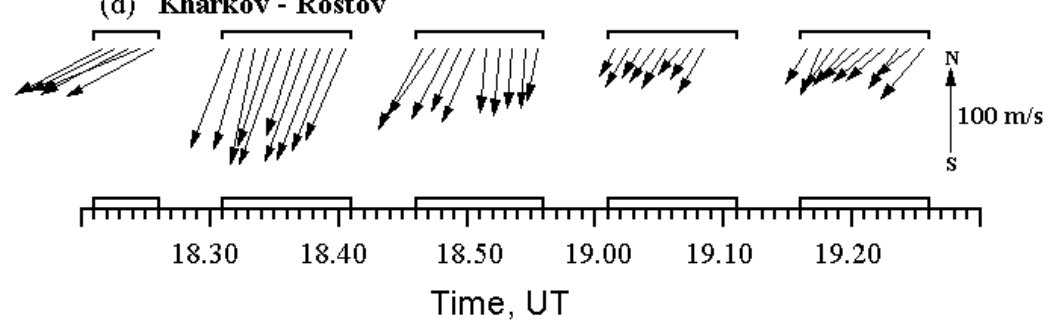

Fig. 10. Median values of Doppler frequency shifts averaged on the 1-min interval for the HF diagnostic signals scattered from AFAIs on the Armavir-Sura-St. Petersburg, Serpukhov-Sura-Kharkov, and Serpukhov (Moscow)-Sura-Rostov paths in the course of the Sura heating experiment on 20 August 2004 (a); simulated vectors of TID phase velocities for the same event using the pair of paths with reception points at St. Petersburg and Rostov (b); the same as in (b) but for St. Petersburg and Kharkov pair paths (c); the same as in (b) and (c) but for Kharkov and Rostov pair paths (d).

have been found. On 19 August, under quiet magnetic conditions, the TIDs with periods of 20 min were traveling in the northeast direction, at speeds between 40 and $80 \mathrm{~m} / \mathrm{s}$. During the experiment on 20 August, conducted under disturbed magnetic conditions, the TIDs were traveling in the southwest direction with higher velocities of $60-150 \mathrm{~m} / \mathrm{s}$. An important point is that two waves with different periods were simultaneously observed, suggesting that two sources of AGWs were involved at the same time.

Taking into account that medium-scale AGWs do not propagate as far as the large-scale waves, one would expect that the AGW source, responsible for the mid-latitude medium-scale TID, was located in the mid-latitude thermosphere, at least during quiet magnetic conditions. Because 
of this, we suggest that the TID with a period of $20 \mathrm{~min}$, observed on 19 August 2004, was induced by a local thermospheric source. The longer the period of an AGW, the further it would propagate. During the experiment on 20 August 2004, under disturbed magnetic conditions, the mid-latitude TIDs with periods of about $45 \mathrm{~min}$ may be the response to an AGW launched from the auroral zone. As shown in Sect. 4, ULF activity can be identified in the Doppler sonogram (see Fig. 7). This is additional evidence for auroral ionospheric sources (Bristow et al., 2004).

\section{Conclusions}

Experimental results from Troms $\emptyset$ and Sura heating experiments have shown that the combination of an HF heaterinduced target and bi-static HF Doppler scatter observations is a valuable method for the identification and study of medium-scale traveling ionospheric disturbances, both at high and mid-latitudes. HF ionospheric modification experiments provide a way of producing the HF heater-induced target by a controlled manner at altitudes where the sensitivity to the TIDs is highest.

Medium-scale traveling ionospheric disturbances with periods of about $23 \mathrm{~min}$ were detected in an auroral sporadic Es-layer in the pre-midnight hours. This point is interesting in itself because the previous studies have been related to TIDs in the F-region of the ionosphere. These TIDs were traveling southward at speeds of $210 \mathrm{~m} / \mathrm{s}$. We suggest that the observed TID was the response to an atmospheric gravity wave launched by the nightside convection-reversal boundary.

Mid-latitude medium-scale TIDs in the ionospheric Fregion have been studied by the use of multi-position bistatic HF Doppler radio scatter observations and simulations. A computational technique has been developed, allowing the reconstruction of the vector of the phase velocities of TIDs from multi-position bi-static HF Doppler scatters. TIDs were observed in evening and pre-midnight hours, under both quiet and disturbed magnetic conditions. During quiet conditions, TIDs with periods of about $20 \mathrm{~min}$ were traveling in the northeast direction at speeds between 40 and $80 \mathrm{~m} / \mathrm{s}$. It is most likely that in this case the AGWs were launched by a local thermospheric source.

Under disturbed magnetic conditions the medium-scale TIDs were traveling in the southwest direction with higher speeds as compared with the magnetically quiet day of about $60-150 \mathrm{~m} / \mathrm{s}$. The observation of different wave periods in the observed TIDs points to the possible existence of multiple gravity waves from different sources. We suggest that the AGW with a period of 45 min was launched by an auroral source in the ionosphere. In addition, the ULF pulsations, which can be seen in the Doppler data at the same time, provide further evidence in support of an ionospheric auroral source.
Acknowledgements. We would like to thank the director and staff of the EISCAT Scientific Association for providing the Troms $\varnothing$ heating experiments in November 2004. EISCAT is an International Association supported by Finland (SA), France (CNRS), Germany (MPG), Japan (NIPR), Norway (NFR), Sweden (NFR) and the United Kingdom (PPARC). The Sura heating campaign in August 2004 was supported by Grant ATM-0000196 from the Atmospheric Science Section of the National Science Foundation. The work has been supported by the INTAS, grant $03-51-5583$ and by the RFBR, grants 04-05-64160, 05-02-16493.

Topical Editor M. Pinnock thanks P. L. Dyson and I. McCrea for their help in evaluating this paper.

\section{References}

Afraimovich, E. L., Kosogorov, E. A., Leonovich, L. A., and Palamartchouk, K. S.: Observation of large-scale traveling ionospheric disturbances of auroral origin by global GPS networks, Earth Planets Space, 52, 669-674, 2000.

Afraimovich, E. L., Vugmeister, B. O., and Kalikhman, A. D.: Comparison of the thermospheric wind dynamics using transionospheric and vertical-incidence radio sounding data, Adv. Space Res., 18, 121-125, 1996.

Afraimovich, E. L.: Interferometer methods in ionospheric radio sounding (in Russian), Nauka, Moskow, 1982.

Blagoveshchenskaya, N. F. and Troshichev, O. A.: Ionospheric phenomena produced by modification experiments, J. Atmos. Terr. Phys., 58, 397-406, 1996.

Blagoveshchenskaya, N. F., Borisova, T. D., Kornienko, V. A., Leyser, T. B., Rietveld, M. T., and Thidé, B.: Artificial fieldaligned irregularities in the nightside auroral ionosphere, Adv. Space Res., doi:10.1016/j.asr.2004.12.008, 2006.

Blagoveshchenskaya, N. F., Chernyshev, M. Yu., and Kornienko, V. A.: Excitation of small-scale waves in the $F$ region of the ionosphere by powerful HF radio waves, J. Atmos. Terr. Phys., 60, 1225-1232, 1998a.

Blagoveshchenskaya, N. F., Kornienko, V. A., Petlenko, A. V., Brekke, A., and Rietveld, M. T.: Geophysical phenomena during an ionospheric modification experiment at Troms $\varnothing$, Ann. Geophys., 16, 1212-1225, 1998b.

Blagoveshchenskaya, N. F., Kornienko, V. A., Brekke, A., Rietveld, M. T., Kosch, M. J., Borisova, T. D., and Krylosov, M. V.: Phenomena observed by HF long-distance tools in the HF modified auroral ionosphere during magnetospheric substorm, Radio Sci., 34, 715-724, 1999.

Borisova, T. D., Blagoveshchenskaya, N. F., Moskvin, I. V., Rietveld, M. T., Kosch, M. J., and Thidé, B.: Doppler shift simulation of scattered HF signals during the Troms $\varnothing$ HF pumping experiment on 16 February, 1996, Ann. Geophys., 20, 1479-1486, 2002 , http://www.ann-geophys.net/20/1479/2002/.

Braude, S. Ya., Megn, A. V., Ryabov, B. P., Sharykin, N. K., and Zhouk, I. N.: Decametric survey of discrete sources in the Northern sky, Astrophys. Space Sci., 54, 3-36, 1978.

Bristow, W. A., Greenwald, R. A., and Samson, J. C.: Identification of high-latitude acoustic gravity wave sources using the Goose Bay HF radar, J. Geophys. Res., 99, 319-331, 1994.

Cheng, K. and Huang, Y. N.: Ionospheric disturbances observed during the period of Mount Pinutubo explosion in June 1991, J. 
Geophys. Res., 97, 16995-17 004, 1992.

Crowley, G. and McCrea, I. W.: A synoptic study of TIDs observed over the UK during the first WAGS campaign, October 10-18, 1985, Radio Sci., 23, 905-917, 1988.

Francis, S. H.: Global propagation of AGWs: a review, J. Atmos. Terr. Phys., 37, 1011-1054, 1975.

Gershman, B. N., Erukhimov, L. M., and Yashin, Yu. Ya.: Wave Phenomena in Ionospheric and Space Plasmas (in Russian), Nauka, Moscow, 1984.

Hocke, K., Schlegel, K., and Kirchengast, G.: Phases and amplitudes of TIDs in the high-latitude $F$ region as observed by EISCAT, J. Atmos. Terr. Phys., 58, 245-255, 1996.

Hunsucker, R. D.: AGWs generated in the high-latitude ionosphere: a review, Rev. Geophys. Space Phys., 20, 293-315, 1982.

Jacobson, A. R. and Carlos, R. S.: Observations of AGWs in the thermosphere following space-shuttle ascent, J. Atmos. Terr. Phys., 56, 525-528, 1994.

Jenkins, G. and Watts, D.: Spectral analysis and its applications, Holden Day, San Fransisco, 1968.

Kirchengast, G., Hocke, K., and Schlegel, K.: Gravity waves determined by modeling of TIDs in ISR measurements, Radio Sci., 30, 1551-1567, 1995.

Rietveld, M. T., Kosch, M. J., Blagoveshchenskaya, N. F., Kornienko, V. A., Leyser, T. B., and Yeoman, T. K.: Ionospheric electron heating, optical emissions, and striations induced by powerful $\mathrm{HF}$ radio waves at high latitudes: Aspect angle dependence, J. Geophys. Res., 108, 1141, doi:10.1029/2002JAA009543, 2003.
Rietveld, M. T., Kohl, H., Kopka, H., and Stubbe, P.: Introduction to ionospheric heating at Troms $\varnothing$ - I. Experimental overview, J. Atmos. Terr. Phys., 55, 577-599, 1993.

Samson, J. C., Greenwald, R. A., Ruohoniemi, J. M., Frey, A., and Baker, K. B.: Goose Bay radar observations of earth-reflected AGWs in the high latitude ionosphere, J. Geophys. Res., 95, 7693-7709, 1990.

Williams, P. J. S. and Virdi, T. S.: EISCAT observations of tidal modes in the lower thermosphere, J. Atmos. Terr. Phys., 51, 569577, 1989.

Williams, P. J. S.: Tides, Atmospheric gravity waves and traveling disturbances in the ionosphere, in: Modern Ionospheric Science, edited by: Kohl, H., Rüster, R., and Schlegel, K., 136-180, 1996.

Williams, P. J. S., Virdi, T. S., Lewis, R. V., Lester, M., McCrea, I. W., and Freeman, K. S. C.: Worldwide Atmospheric Gravity wave Study in the European sector 1985-90, J. Atmos. Terr. Phys., 55, 683-696, 1993.

Yampolski, Y. M., Beley, V. S., Kasheev, S. B., Koloskov, A. V., Somov, V. G., Hysell, D. L., Isham, B., and Kelley, M. C.: Bistatic HF radar diagnostic induced field-aligned irregularities, J. Geophys. Res., 102(A4), 7461-7467, 1997.

Yeh, K. C. and Liu, C. H.: AGWs in the upper atmosphere, Rev. Geophys. Space Sci., 12, 193-216, 1974. 